\title{
Spherical Object Reconstruction Using Simplex Meshes from Sparse Data
}

\author{
Pavel Matula and David Svoboda \\ Laboratory of Optical Microscopy \\ Faculty of Informatics, Masaryk University \\ Botanická 68a, CZ-602 00 Brno, Czech Republic \\ \{pam,xsvobod2\}@fi.muni.cz
}

\begin{abstract}
A new method for spherical object reconstruction based on deformation of star-shaped simplex meshes has been developed in our laboratory and published recently. The method can handle volumetric as well as three-dimensional range data and is easy to use and relatively fast. The method, however, can yield wrong results for sparse data. The goal of this paper is to describe a modification of the method that is suitable also for sparse data. The performance of the proposed modification is demonstrated on real biomedical data.
\end{abstract}

Keywords: Spherical objects, object reconstruction, deformable models, sparse data, simplex mesh, volumetric image segmentation.

\section{Introduction}

Spherical object reconstruction is of great importance, especially in the field of cell biology in the research of 3D organisation of the human genome, since both cells and cell nuclei mostly have the shape of a deformed sphere. Biological applications very often require processing of a large number of cells or cell nuclei to obtain statistically significant results. One of the key issues in this field is to have a good 3D model of object boundaries. Therefore fast, reliable and precise procedure for the automatic image analysis and objects reconstruction is needed.

Good reconstruction methods in this field must yield good results also for sparse data. The reason for this is that the biochemical visualisation of nuclear envelope using standard methods of molecular biology and cytology is not always completely successful and only several parts of nuclear surface can clearly be visible in volumetric image data. Therefore even the best image analysis technique cannot determine, in principle, the whole nuclear envelope.

Many techniques for the reconstruction of cell nuclei are based on thresholding [14|15]. In this case the nuclei are represented as a set of voxels and a boundary representation can be produced by boundary tracking algorithms (see [7]). Isosurfacing methods based on marching cubes algorithm [8] are also used 64. However, these methods do not handle missing and noisy data and can be hardly used for reconstruction from sparse input data, because they make no assumption about the shape to recover. 
Deformable modelling [13,12] is suitable for the reconstruction of objects from incomplete and noisy data because it can exploit an apriori knowledge about the reconstructed shape. There are several existing frameworks for deformable models, but a common approach is to consider the deformation as an iterative process minimising a required trade-off between an internal energy controlling apriori known shape qualities of the model and an external force controlling the closeness of fit.

Three-dimensional objects of an arbitrary shape and topology can be reconstructed using Delingette general algorithm, which is based on deformation of simplex meshes [3]. The algorithm works for both volumetric and range data. One of the advantages of simplex meshes is that their deformation can be performed in a simple and efficient manner. Simple deformation is possible at the expense of not having a global functional for guiding the minimisation. Certain apriori knowledge about the shape of an unknown object have to be involved in the deformation process only by means of constraints on the local shape of the simplex mesh.

The idea of the general algorithm was exploited during the design of a new method for spherical object reconstruction [11]. Star-shaped simplex meshes are considered for object representation instead of general simplex meshes. Therefore, a deformation scheme can be simplified and the star-shaped method is faster and more stable (in the meaning of convergence for more minimisation parameters) 9].

This paper discusses the usage of both the general and star-shaped method for spherical object reconstruction from sparse data. First, the necessary background of the methods is given in Sect. 2. In Sect. 3. the usage of the methods on sparse data is discussed and a modification of the methods is proposed to make it more suitable for spherical object reconstruction from sparse data. Section 4 presents an application example of the modified star-shaped method.

\section{Method Background}

This section reviews the basics of reconstructions using simplex meshes and star-shaped simplex meshes.

\subsection{Simplex Meshes}

The surface of an object can be represented using a simplex mesh [2/3]. Simplex mesh is a structure consisting of vertices and edges. The vertices are points in 3D space. Every edge connects two distinct vertices. The shorter the edges are the more details of the surface can be modelled. Important property of a simplex mesh is that each vertex has exactly three neighbouring vertices connected via edges (see Fig. 6). A simplex mesh is called star-shaped (has the shape of a star) if a point exists inside the mesh such that any ray going from the point intersects the mesh only once. The set of such points inside the mesh is called kernel of star-shaped simplex mesh.

Thanks to the property of three neighbours, the following definitions can be provided. Tangent plane at a vertex is given by its three neighbours. Normal 
vector at a vertex is equal to the normal vector of the tangent plane. Local shape of the simplex mesh can be controlled by means of a simplex angle. The simplex angle at a vertex is related to the local mean curvature of the surface at this vertex. Only one sphere can be circumscribed to the vertex and its three neighbours. This sphere can be seen as an approximation of the local shape near the vertex. The simplex angle expresses how much this sphere is elevated upon or sunken bellow the tangent plane. The simplex angle is invariant to the position of the vertex on the sphere and to the position of the three neighbours on a circle circumscribed to these three points. The simplex angle is also invariant to translation, rotation and scale transformations [2].

\subsection{Deformation of Simplex Meshes}

Law of Motion. All vertices of a simplex mesh are considered as a physical mass submitted to a Newtonian law of motion including internal and external forces. The discrete formula is [3]:

$$
P_{i}^{t+1}=P_{i}^{t}+(1-\gamma)\left(P_{i}^{t}-P_{i}^{t-1}\right)+\alpha \boldsymbol{F}_{\text {int }}+\beta \boldsymbol{F}_{\text {ext }},
$$

where $P_{i}^{t}$ is a position of $i$-th vertex in time $t$. Internal force $\boldsymbol{F}_{\text {int }}$ and external force $\boldsymbol{F}_{\text {ext }}$ are computed at time $t$ and are defined further. Real parameter $\gamma$ is the damping factor. Real parameters $\alpha$ and $\beta$ must belong to a given interval to guarantee a stable scheme and their ratio expresses the trade-off between influence of internal and external forces, i.e. between required local shape of the mesh and the closeness of fit.

All forces deforming a star-shaped simplex mesh are acting only along rays called deformational rays from a proper point in the kernel of an initial simplex mesh (usually the centre of an initial ellipsoid is used). The point is called deformational centre. In this way the star-shaped quality is preserved during the deformation process. The general and star-shaped methods differ in the definition of the internal and external force.

Internal Force. The internal force of Delingette's general algorithm is defined as the composition of a tangential force and a normal force. The goal of the tangential force is to control the vertex position with respect to its three neighbours in the tangent plane, i.e. uniformly spread the vertices of the final mesh.

The normal force is acting in order to change the local mean curvature at a vertex to the required local shape. The requirements are expressed by means of a reference simplex angle. The reference simplex angle can be determined in four ways [3]. However, only two of them are valuable for spherical object reconstruction:

shape constraint. The reference simplex angle $\tilde{\varphi}_{i}$ is equal to a constant value $\varphi_{i}^{0}$. The value can be, for example, computed from an initial ellipsoid or sphere. In this way the internal force can keep the spherical shape of the mesh. 
$\boldsymbol{C}^{\mathbf{2}}$ constraint. The reference simplex angle $\tilde{\varphi}_{i}$ is computed as an average of the simplex angles at neighbouring vertices:

$$
\tilde{\varphi}_{i}=\frac{1}{n} \sum_{j \in Q^{s_{i}}\left(P_{i}\right)} \varphi_{j},
$$

where $Q^{s_{i}}\left(P_{i}\right)$ is the set of all vertices, which are reachable in at most $s_{i}$ steps via edges from the vertex $P_{i}$ (neighbourhood with size $s_{i}$ ). Number $n$ is the cardinality of this set. The neighbourhood size $s_{i}$ corresponds intuitively to the notion of rigidity, or deformation scale.

The tangential force is not needed for the deformation of star-shaped simplex meshes. A deformational ray plays the role of this force. The internal force of the star-shaped method behaves only like the normal force of the general method. The difference is only in the direction of activity and in the way of computation. The role of both forces is the same [11].

External Force. The external force for general simplex meshes can be defined for both volumetric and range data [3. In all cases, the external force is directed along the normal direction $\boldsymbol{n}_{i}$ at the vertex $P_{i}$, where the force is applied. For every vertex $P_{i}$ of the mesh, the closest point $Q_{i}$ is searched in a given scope and the force $\boldsymbol{F}_{\text {ext }}$ is then computed as

$$
\boldsymbol{F}_{\text {ext }}=\left(\left(Q_{i}-P_{i}\right) \cdot \boldsymbol{n}_{i}\right) \boldsymbol{n}_{i},
$$

if $\left(Q_{i}-P_{i}\right) \cdot \boldsymbol{n}_{i}<\tilde{G}$, where $\tilde{G}$ is an user defined parameter called gravitation limit. The external force is set to $\mathbf{0}$ otherwise. The force is computed as the projection of the vector $\left(Q_{i}-P_{i}\right)$ in the normal direction. Notice that if there is no data point in the vicinity of a mesh vertex (defined by the gravitation limit) then no external force influences the vertex and the vertex is only submitted to internal (regularization) force.

The force $\boldsymbol{F}_{\text {ext }}$ is defined similarly for star-shaped simplex meshes. The force is not, however, projected in the normal direction of the vertex, but onto the corresponding deformational ray [119.

\subsection{Reconstruction Algorithm}

The general reconstruction algorithm works in two stages. First, the initialisation stage creates a simplex mesh in the vicinity of the data and then, after a few iterations, the mesh topology can be modified. Finally, an iterative refinement algorithm decreases the distance of the mesh from the data while preserving high geometric and topological quality.

During the first stage Delingette recommends to use $C^{2}$ constraint for internal force computation and set the neighbourhood size $s_{i}$ to high values $(\approx 10)$ and $\beta$ to low values $(\approx 0.1)$ in order to obtain smooth and large scale deformations of the mesh. The gravitation limit $\tilde{G}$ is recommended to be set to relatively large value (up to $20 \%$ of the overall size of the dataset) in order to allow large deformations of the mesh. 

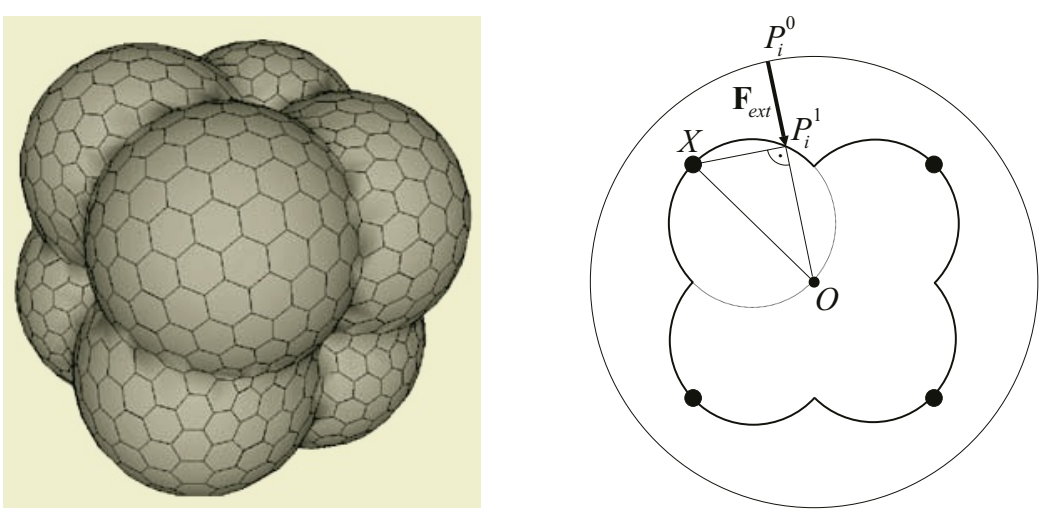

Fig. 1. Reconstruction of the cube vertices. One iteration was performed while only external forces were active. Thanks to the perpendicular projection the mesh vertices lie on spheres given by the cube vertices and the centre of the initial mesh. The result is the same for both the general and star-shaped methods.

The second stage is recommended to be performed with parameters set as follows $s_{i} \approx 1, \beta \approx 0.5$, and $\tilde{G}<8 \%$. The deformation is stopped when the change of the mesh is not significant (is less then a predefined constant $\varepsilon$ ). Remaining parameters are recommended to be set during the whole reconstruction process as follows $\alpha=0.5, \gamma=0.65$.

The reconstruction algorithm based on star-shaped simplex meshes works also in two stages. However, the initialisation phase is usually performed in only one iteration. The iteration is done with parameters $\alpha=0, \beta=1$, i.e. only external forces are active while gravitation limit is large. Therefore the mesh settles onto the input data in a given scope and interpolates them. The task for the refinement stage, where the internal forces are employed and the influence of the external forces is reduced, is to smooth the mesh.

\section{Reconstruction from Sparse Data}

\subsection{The Problem}

Both the general and the star-shaped methods can fail in case of sparse data. The source of the problem of the methods can be demonstrated on a simple example of sparse data reconstruction described bellow. Let the input data be eight vertices of a cube with unit edges (vertices have coordinates $\left[ \pm \frac{1}{2}, \pm \frac{1}{2}, \pm \frac{1}{2}\right]$ ). The initial mesh have a shape of the unit radius sphere with centre in the origin $O$. If one iteration according to (1) with parameters $\gamma=1, \alpha=0, \beta=1$, and $\tilde{G}=2$ is performed, i.e. only external forces are active and are strong, then the mesh have the shape as in Fig. 11 This shape is a result of the definition of external force. Perpendicular projection of the nearest data point $X$ is computed and therefore the mesh vertices lie on the circles with diameter $X O$. Notice that the normal directions coincide with the direction of the deformation rays. The behaviour of both the general and star-shaped method is the same in this example. 
Evidently, the definition of the external force is in conflict with an apriori knowledge about the spherical shape. This problem especially affects sparse data reconstruction during the initialisation stage, because the gravitation limit is typically large. The bad initialisation stage can significantly slow down the whole reconstruction process.

However, the refinement stage is affected as well. The gravitation limit must be sufficiently high during the minimisation in order that the external forces can keep the mesh near the data points. Therefore protuberance occur close to the data points and the surface is consequently underestimated. See Fig. 2
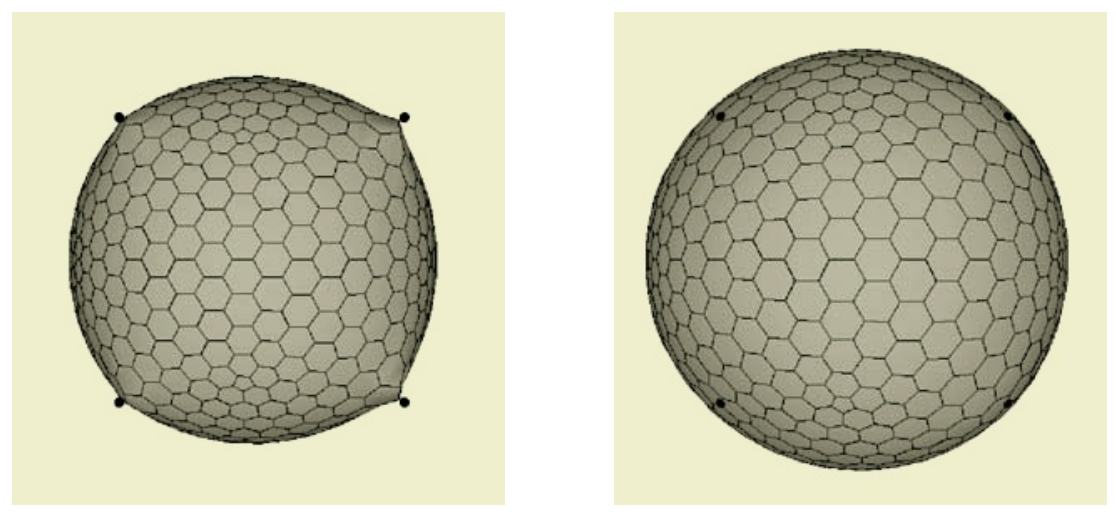

Fig. 2. (left) The mesh was computed by the general method with parameter setting $\beta_{i}=0.1, s_{i}=10, \alpha_{i}=0.5, \gamma=0.8$ during the initialisation stage (10 iterations, $C^{2}$ constraint) and $\alpha_{i}=\beta_{i}=0.5, \gamma=0.8, \tilde{G}=0.1$ during the refinement stage (250 iterations, shape constraint). The initial mesh had a shape of the unit sphere with centre in the origin. Eight data points of coordinates $\left[ \pm \frac{1}{2}, \pm \frac{1}{2}, \pm \frac{1}{2}\right]$ were shifted to the left by the vector $[0,0.14,0]$. Perpendicular projection was applied for external force computation. Notice that protuberance occur close to the data points and the surface is underestimated. (right) The mesh was computed by the star-shaped method, which was modified according to Section 3.2 i.e. spherical projection was applied for external force computation. The initial mesh and data points were the same as on the left. Refinement stage was performed (250 iterations) with parameters $\alpha_{i}=\beta_{i}=0.5$, $\gamma=0.8, \tilde{G}=0.1$.

\subsection{The Solution}

We suggest using a spherical projection instead of the perpendicular projection for spherical object reconstruction from sparse data as a solution to this problem. Let $O$ be a deformational centre (see Sect. 2.2) and $X$ be the closest point of vertex $P_{i}$. The external force is redefined

$$
\boldsymbol{F}_{\text {ext }}=\left(P_{i}-O\right)\left(1-\frac{\|X-O\|}{\left\|P_{i}-O\right\|}\right) .
$$

The proposed solution is illustrated in Fig. 3. The novel external force definition for spherical object reconstruction can also be used in the general method. 

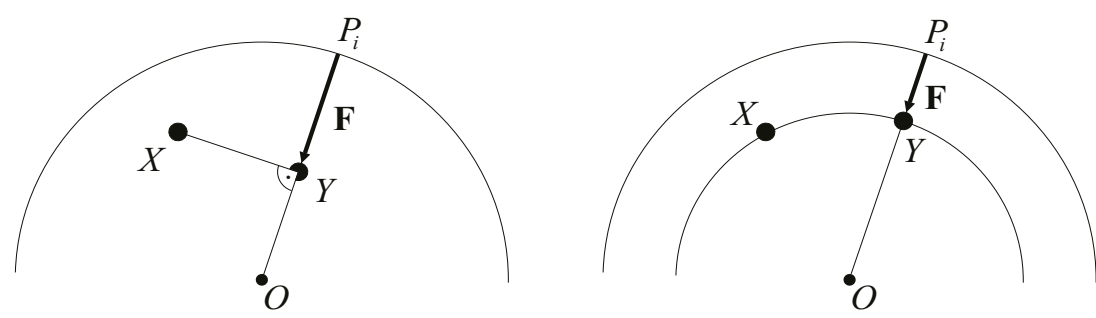

Fig. 3. Figure illustrates the proposed solution of the problem from Fig. 1. Spherical projection is used in the external force computation instead of the perpendicular projection.

Only the point $O$ must be defined. One of the possible definitions is to take the point $O$ equal to the centre of the initial ellipsoidal mesh. It is assumed that the mesh does not move much and only its shape and size are changed during the deformation. The spherical projection is computed in the normal direction of mesh points. In both cases the gravitation limit $(\tilde{G})$ for the maximal value of an external force is applied.

Notice that considering this redefinition, the reconstruction of cube vertices in the first example above reaches the sphere circumscribed to the cube already after the first iteration.

\section{Application Example}

Both the general method and the star-shaped method with the proposed external force redefinition can be applied for cell nucleus reconstruction from sparse data. Only the results of the star-shaped method are presented because the results do not differ significantly for the general method. The test volumetric images of $3 \mathrm{D}$ fixed cell nuclei obtained from the stabilised cell line of human colon adenocarcinoma HT-29 (the nuclear envelope was visualised using Lamin B) were acquired by a confocal microscope. The typical size of the data was $140 \times 140 \times 40$ voxels. The resolution was $0.124 \mu \mathrm{m} /$ voxel in the lateral and $0.3 \mu \mathrm{m} /$ voxel in the axial direction. The images were manually cropped from large volumetric images. One of the test images is depicted in Fig. 4 (left column) as two cross-sections through the data.

The fractions of the nuclear envelope visible in images were automatically extracted by image analysis methods (Gaussian smoothing was followed by thresholding with a suitable threshold). Results of this step are presented in Fig. 4 (middle column). The extracted voxels were then considered as a point set. The point set was approximated by an ellipsoid in a least squares manner. The ellipsoid superimposed onto the image data is presented in Fig. 4 (right column). In the last step before running the deformation process the ellipsoid was covered with an initial mesh.

The mesh was deformed according to the reconstruction algorithm for starshaped simplex meshes (Sect. 2.3). The external force was computed by the 

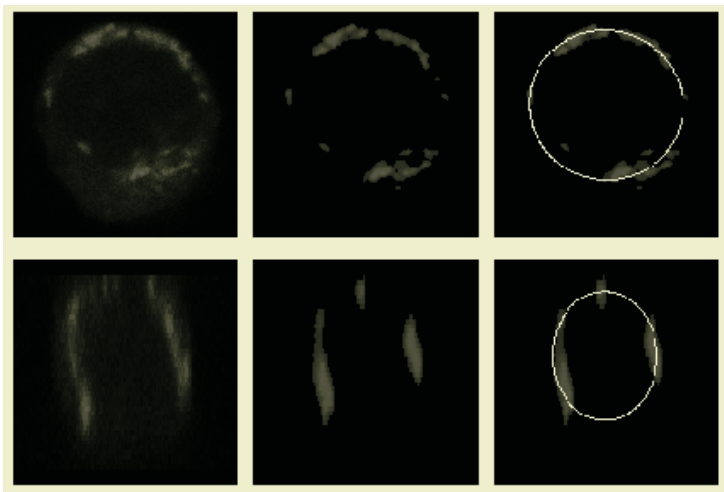

Fig. 4. Input data. The upper row shows in sequence from left to right: $x y$ slice $(z=20)$ of the raw 3D input image, object boundary points extracted from this slice, and projection of an initial ellipsoid fitted to the boundary points onto the $20^{\text {th }} x y$ slice; the lower row corresponds to the same data, only $x z$ slice $(y=50)$ is shown.
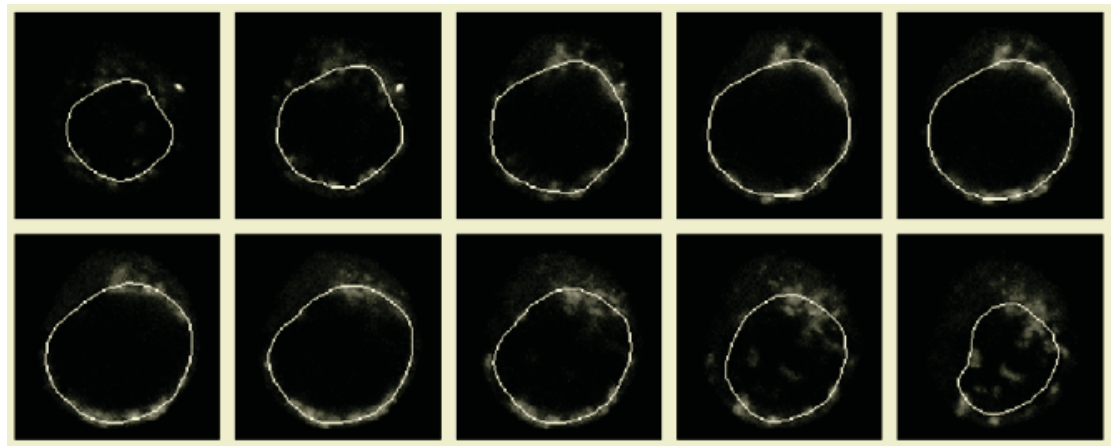

Fig. 5. The final mesh is projected onto the raw input data. The upper row shows $x y$ slices at $z=1,4,7,10,13$. The lower row shows $x y$ slices at $z=15,18,21,24,27$.

proposed definition (3). The best results were obtained for the following parameters. The initialisation stage (one iteration) was run with parameters $\alpha=0$, $\beta=1, \tilde{G} \approx 30 \%$ of the dataset size. The refinement stage was run with parameters $\alpha=0.8, \beta=0.2, \gamma=0.8, \tilde{G} \approx 5 \%$ of the dataset size. Projection of the mesh onto the raw data after 100 iterations is shown in Fig. 5 . The shape of the final mesh and its relation to the extracted voxels is shown in Fig. 6.

\section{Conclusion}

The problem of the applicability of deformable simplex meshes for spherical object reconstruction from sparse data was studied. We have discovered that the general [3] and the star-shaped 119] reconstruction algorithms can yield poor results on sparse data. A redefinition of external forces suitable for spherical 

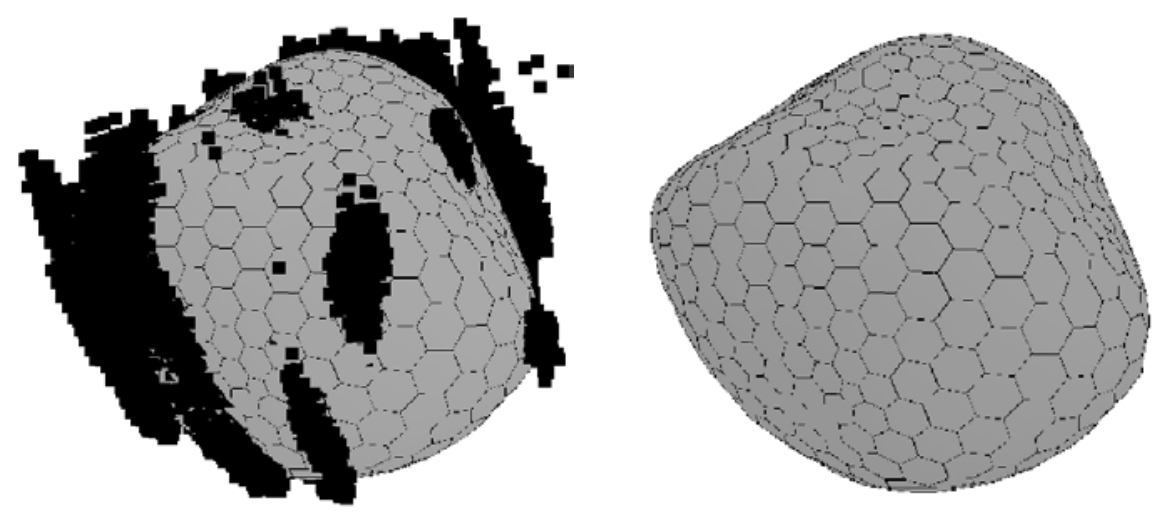

Fig. 6. (Left) Range data extracted from the input volumetric image and the fitted mesh. (Right) The final mesh alone.

object reconstruction was proposed in this paper. The method was tested on real biological data acquired by a confocal microscope. The proposed modification increases the speed of the reconstruction process (the number of iterations is reduced) and the final meshes seem to be more natural. The assumption about the spherical shape of the reconstructed objects is expoited by the novel external force definition in a natural way.

\section{Acknowledgements}

This work was supported by the Ministry of Education of the Czech Republic (Project No. MSM-143300002) and by the Academy of Sciences of the Czech Republic (Grants No. S5004010 and No. B5004102). We thank Irena Koutná for providing the image data. We also thank both reviewers for their useful comments.

\section{References}

1. C. O. de Solórzano, E. García Rodriguez, A. Jones, D. Pinkel, J. W. Gray, D. Sudar, and S. J. Lockett. Segmentation of confocal microscope images of cell nuclei in thick tissue sections. Journal of Microscopy, 193:212-226, 1999.

2. H. Delingette. Simplex meshes: a general representation for 3D shape reconstruction. Technical Report 2214, INRIA, France, 1994.

3. H. Delingette. General object reconstruction based on simplex meshes. International Journal of Computer Vision, 32(2):111-146, 1999.

4. F. Guilak. Volume and surface area measurement of viable chondrocytes in situ using geometric modelling of serial confocal sections. Journal of Microscopy, 173(3):245-256, 1994.

5. M. Kozubek, S. Kozubek, E. Lukášová, A. Marečková, E. Bártová, M. Skalníková, and A. Jergová. High-resolution cytometry of FISH dots in interphase cell nuclei. Cytometry, 36:279-293, 1999. 
6. L. Kubínová, J. Janáček, F. Guilak, and Z. Opatrný. Comparison of several digital and stereological methods for estimating surface area and volume of cell studied by confocal microscopy. Cytometry, 36:85-95, 1999.

7. G. Lohmann. Volumetric Image Analysis. John Wiley \& Sons, Inc. and B. G. Teubner, 1998.

8. W. E. Lorensen and H. E. Cline. Marching cubes: A high resolution 3D surface construction algorithm. In Computer Graphics (SIGGRAPH '87), volume 21, pages 163-169, 1987.

9. Pavel Matula. Effectivity of spherical object reconstruction using star-shaped simplex meshes. In Guido M. Cortelazzo and Concettina Guerra, editors, 1st International Symposium on 3D Data Processing Visualisation and Transmission, pages 794-799, Padova, Italy, June 2002. IEEE Computer Society.

10. Pavel Matula. Threedimensional object reconstruction and its application in cytometry. PhD thesis, Faculty of Informatics, Masaryk University, Brno, september 2002. in czech.

11. Pavel Matula and D. Svoboda. Spherical object reconstruction using star-shaped simplex meshes. In Mário Figueiredo, Joasine Zerubia, and Anil K. Jain, editors, Energy Minimization Methods in Computer Vision and Pattern Recognition, volume 2134 of LNCS, pages 608-620, Sophia Antipolis, France, September 2001. Springer Verlag.

12. T. McInerney and D. Terzopoulos. Deformable models in medical image analysis: A survey. Medical Image Analysis, 1(2):91-108, 1996.

13. J. Montagnat, H. Delingette, and N. Ayache. A review of deformable surfaces: topology, geometry and deformation. Image and Vision Computing, 19:1023-1040, 2001.

14. H. Netten, I. T. Young, L. J. Van Vliet, H. J. Tanke, H. Vrolijk, and W. C. R. Sloos. FISH and chips: automation of fluorescent dot counting in interphase cell nuclei. Cytometry, 28:1-10, 1997. 